\title{
Alphonse de Lamartine oder die Lyrik der Einsamkeit
}

Es gibt - wie wir sahen - viele Wege, die hinein in die Romantik führen, aber auch einige ganz wunderbare Pfade, auf denen man wieder aus ihrem Bannkreis heraustritt. Ich möchte Ihnen dies gerne auf dem Gebiet der Lyrik vorführen und will dabei zunächst tief in der Romantik beginnen. Es ist daher an der Zeit, Ihnen eines der großen, sicherlich auch berühmtesten und meistzitierten Gedichte dieser Epoche vorzustellen, das aus der Feder des Franzosen Alphonse de Lamartine stammt und jenen Vers enthält, der in gewisser Weise - wir haben es schon zuvor erwähnt - geradezu als Definition des Verhältnisses zwischen Individuum, Gesellschaft und Natur gelten darf. Dieses triadische Verhältnis wird - wie könnte es anders sein? - vermittelt über eine romantische Liebeskonzeption, die in ihrem Absolutheitsanspruch hinter dem geliebten Menschen alles andere, ja die ganze Welt deutlich zurücktreten lässt: „Ein einzig Wesen fehlt, und alles ist entvölkert.“ Doch wer war der Verfasser dieses wohl berühmtesten Verses der nicht nur französischen Romantik, der in vielerlei Varianten von den Schriftstellerinnen und Schriftstellern der Literaturen der Welt in den unterschiedlichsten Sprachen und Varianten zitiert und weitergeführt, in nachromantischer Zeit dann aber auch nicht selten verhohnepiepelt wurde?

Alphonse Marie Louis Prat de Lamartine wurde am 21. Oktober 1790 im französischen Mâcon geboren und starb am 28. Februar 1869 in Paris. Er gehörte zu jenen Romantikern, die sich nicht nur einen Namen in der Literatur und bisweilen in den Schönen Künsten, sondern auch in der Politik machten und darf in diesem Sinne durchaus in seiner Bedeutung mit Chateaubriand verglichen werden, bei dem wir diese Kombination ausführlich analysierten.

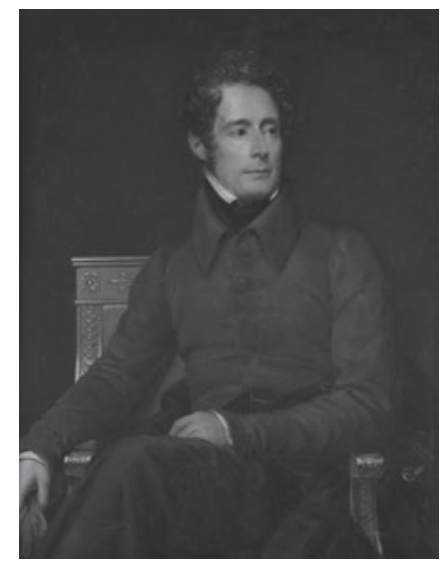

Abb. 63: Alphonse de Lamartine

(Mâcon, 1790 - Paris, 1869).

2 Open Access. (c) 2021 Ottmar Ette, publiziert von De Gruyter. (cc)BY-NC-ND Dieses Werk ist lizensiert unter einer Creative Commons Namensnennung - Nicht-kommerziell - Keine Bearbeitung 4.0 International Lizenz. https://doi.org/10.1515/9783110703443-028 
Alphonse de Lamartine stammt aus einer royalistischen Familie des Landadels und wuchs in Milly in einer höchst provinziellen Umgebung auf. Als einzigem Sohn sollte ihm das väterliche Erbe zufallen; er wurde konservativ und katholisch erzogen, besuchte ein Internat im nahegelegenen Lyon, von wo der Zwölfjährige ausriss, und wechselte später in ein ehemaliges Jesuitenkolleg in Belley. Erste bleibende, künstlerisch bedeutende Eindrücke hinterließ in den Jahren 1811 und 1812 die Reise in ein von napoleonischen Truppen besetztes Italien; Eindrücke, die Lamartine auch in einem späteren Roman verarbeitete. 1812 wurde er zum Bürgermeister von Milly und kam erstmals nach Paris. Eine eingeschlagene Offizierslaufbahn, während der er sich zu Zeiten von Napoleons Niedergangs vor dem Schlimmsten bewahren konnte, musste er aus Gesundheitsgründen aufgeben. 1816 lernte er bei einem Kuraufenthalt in Aix-les-Bains Julie Charles kennen. Er verliebte sich in sie und folgte ihr nach Paris, wo er in ihrem Salon verkehrte. Die Liebe zu ihr und ihren überraschenden Tod im Jahr 1817 verarbeitete Lamartine in seinen frühen Gedichten, wobei er die tuberkulosekranke junge Frau und ihr Ableben in intensiven Landschaftsbildern zelebrierte.

Doch schon im Jahr 1820 heiratete er die Engländerin Mary-Anne Birch, die er ein Jahr zuvor kennengelernt hatte. Ab 1817 war Lamartine im diplomatischen Dienst tätig, der ihn in den zwanziger Jahren wiederholt nach Italien führte. Nach einer ernsthaften Erkrankung näherte er sich wieder jener Frömmigkeit der Jugendjahre an, die in seine Gedichte Eingang fand. Auch später stand er den Sozialaussagen der katholischen Kirche nahe. Im März des Jahres 1820 veröffentlichte er seine Gedichtsammlung Méditations poétiques, in denen der Einfluss Rousseaus und der Frühromantik, aber auch der englischen Lyrik unverkennbar waren. Der schmale Gedichtband hatte von Beginn an einen gewaltigen Erfolg und machte den Namen Lamartine schlagartig berühmt. Seine schriftstellerische Laufbahn als Dichter begann, die ihn zehn Jahre später - nach einem ersten vergeblichen Anlauf - zu einem Mitglied der Académie Française und damit zu einem der ,Unsterblichen‘ machen sollte.

Mit der französischen Julirevolution quittierte Lamartine den diplomatischen Dienst, da er den ,Bürgerkönig Louis-Philippe als rechtmäßigen König nicht anerkannte. So nahm in den dreißiger Jahren die Politik in seinem Leben immer breiteren Raum ein; bereits seit 1833 war der einstige Landadelige französischer Abgeordneter. Zuvor allerdings hatte er mit seiner Familie eine Orientreise angetreten, über die er den für die Zeit typisch orientalisierenden Band Voyage en Orient (1835) publizierte. Nach seiner Rückkehr nahmen politische Aktivitäten zu: Er distanzierte sich als Adeliger von Louis-Philippe und war schließlich am Sturz der Regierung beteiligt. Zugleich war er mit seinem 1830 erschienenen Gedichtsband Harmonies poétiques et religieuses endgültig zu einem der Anführer der jungen 
romantischen Schule in Frankreich aufgestiegen. Literatur und Politik gingen bei Lamartine Hand in Hand.

Der Verfasser des später zu besprechenden Gedichts L'Isolement stieg in der Folge zu einer der prägenden politischen Figuren Frankreichs auf, wurde 1848 für kurze Zeit Außenminister und de facto Führer der eingesetzten provisorischen Regierung; doch löste ihn bereits am 24. Juni Louis-Eugène Cavaignac ab. Entscheidend war, dass er zwar als oppositioneller Republikaner zu einem der gefürchtetsten politischen Redner Frankreichs aufgestiegen war, jedoch bei den Präsidentschaftswahlen Ende 1848 deutlich Charles Louis Napoléon Bonaparte unterlag, dem späteren Napoleon III. Nach seinem erzwungenen Rückzug aus der Politik infolge des Staatsstreichs von 1851 veröffentlichte Lamartine - nach seiner achtbändigen Histoire des Girondins (1847) - vor allem historische Werke wie die Histoire de la Restauration (1851-1853), seinen Cours familier de la littérature in achtundzwanzig Bänden sowie autobiographische Erzählungen und Episoden. Letztere konnten ähnlich wie sein Roman Geneviève, histoire d'une servante nicht mehr an die Erfolge seiner frühen Dichtungen anknüpfen. Alphonse de Lamartine gilt gleichwohl - gerade auch durch die Vereinigung von Literatur und Politik in seiner Gestalt - bis heute als einer der großen Vertreter der Romantik in Frankreich.

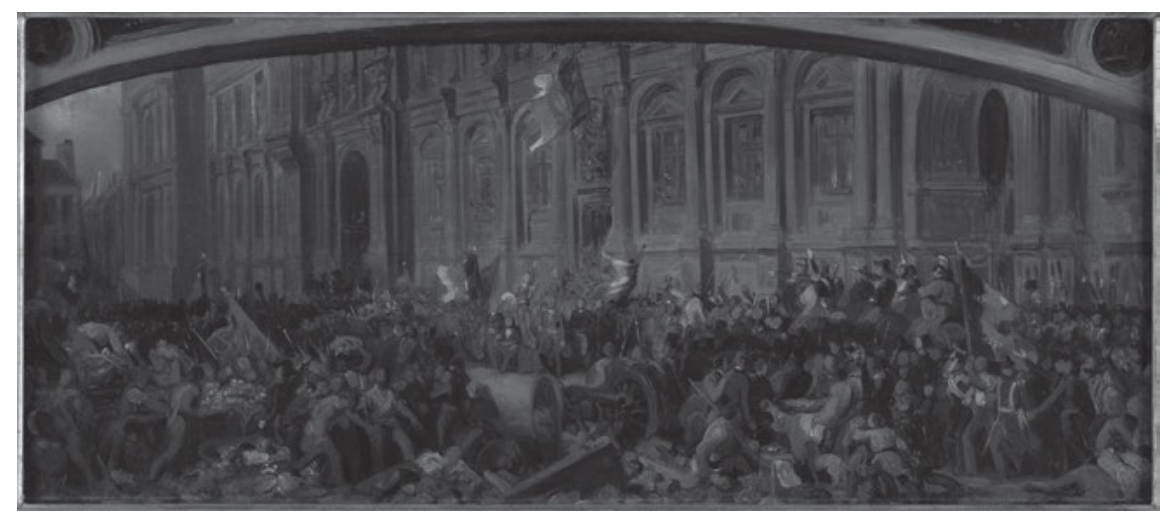

Abb. 64: „Lamartine repoussant le drapeau rouge à l'Hôtel de Ville, le 25 février 1848“, Öl auf Leinwand von Henri-Félix-Emmanuel Philippoteaux, circa 1848.

Sein dichterischer Ruhm gründet sich auf seine frühe Lyrik, die Méditations poétiques von 1820, die rasch in die verschiedensten europäischen Sprachen übersetzt wurden und in deutscher Übersetzung 1826 erschienen, sowie die Nouvelles méditations poétiques von 1823, die ebenfalls im selben Jahr auch auf Deutsch publiziert wurden. Mit diesen Gedichtbänden führte er einen für die französische 
Dichtung neuartigen Ton der Meditation ein, der bis zu diesem Zeitpunkt auf die Philosophie und die Theologie beschränkt gewesen war. Der Dichter aus Mâcon avancierte damit zum lyrischen Wortführer der Romantik, obwohl er sich von Gruppenbildungen stets distanziert hielt.

In geradezu ,klassischer“ Weise setzte sich Lamartine mit dem „mal du siècle“ auseinander, der Melancholie oder Schwermut, meditierte aber auch über Einsamkeit, Liebesschmerz und Vergänglichkeit alles Irdischen. An romantische Autoren wie Lord Byron oder den ihm persönlich bekannten Chateaubriand anknüpfend, reduzierte Lamartine die melancholische Haltung zu einer gedämpften Trauer, die weder in der Religion noch in der Liebe einen festen Halt findet. Auch der Verlust lieber Menschen, wie etwa seiner zehnjährigen Tochter, die auf der gemeinsamen Orientreise erkrankte und starb, bildet einen Themenbereich, den Lamartines dichterische Kunst bearbeitete. Seine späteren Gedichtsammlungen besitzen nicht mehr die Unsicherheit der frühen lyrischen Schöpfungen; denn in ihnen zeigte sich nun stärker die Glaubenssicherheit des Poeten. Sie wurden zwar rasch in verschiedene Sprachen übertragen, konnten beim Publikum aber nicht mehr denselben großen Erfolg verzeichnen. Seine geplanten großen epischen Entwürfe blieben Stückwerk und scheiterten; Lamartine führte sie nicht zu Ende.

In kaum einem anderen Gedicht ist die romantische Vereinzelung des Individuums, die gesellschaftliche Dimension dieser Isolierung, aber auch die Thematik und das Theorem der Einsamkeit so eindringlich in seine ästhetischen und historischen Kontexte gestellt worden wie bei Lamartine. Im Übrigen wird bei einem Vergleich zwischen Leopardi und Lamartine auch schnell deutlich, wie intensiv der interkulturelle Austausch zwischen den verschiedenen europäischen Ländern und National-Literaturen tatsächlich funktionierte. Nicht umsonst war Alphonse de Lamartine seit seiner frühen Reise nach Italien der Literatur des südlichen, vor seiner Nationenbildung stehenden Nachbarlandes sehr zugetan:

Einsamkeit

Oft auf des Berges Hang, in alter Eichen Schatten, In Trauer lieg' ich still, derweil die Sonne sinkt;

Der Zufall führt den Blick durch Felder und durch Matten, Wo wechselvoller Reiz mir zu den Füßen winkt.

Dort tobt der schnelle Fluß mit seiner Wellen Schaume

Er krümmt sich, er versenkt sich in die dunkle Fern'; Hier dehnt der stille See sein Wasser als im Traume, Wo aus dem tiefen Blau sich erhebt der Abendstern. 
Die Gipfel, die den Wald zur düstern Krone tragen, Grüßt noch das Abendrot mit seinem letzten Strahl, Der Schatten Königinn steigt auf dem dunst'gen Wagen, Und färbt den Horizont am runden Saume fahl.

Und mittlerweile schwingt, vom alten Turm gesendet, Herüber durch die Luft sich frommer Töne Klang;

Der Wandrer steht und horcht: des Dorfes Glocke spendet Ins letzte Tagsgeräusch den heiligen Gesang.

Ach! Aber meinen Sinn rührt weder Reiz noch Wonne Bei diesem holden Bild; mein Herz bleibt unentbrannt: Ein Schatte geh' ich um, und der Lebend'gen Sonne Erwärmt die Toten nicht: so schau' ich auf das Land.

Umsonst trag' ich den Blick von Hügel hin zu Hügel, Zum Niedergang vom West, vom Mittag hin zum Nord; Den ungemessnen Raum durchstreift des Geistes Flügel; Doch sprech' ich: Meiner harrt das Glück an keinem Ort.

Was sollt ihr Thäler mir, ihr Hütten, ihr Paläste? Entflogen ist der Reiz, was will der leere Schwall? Du Strom, du Fels, du Hain! Ihr Oeden - mir das Beste! Euch fehlt ein Wesen nur - entvölkert seid ihr all!

Mit unbewegtem Blick ich auf zur Sonne sehe, Ob ihre Wandrung sie beginnen, enden mag; Ob sie umwölkt, ob klar, auf oder nieder gehe: Was gilt die Sonne mir? ich hoffe nichts vom Tag.

Und dürft' ich mit ihr zieh'n hoch über Berg' und Thale, Nur Oede würd' ich schaun und ringsum Wüsteney'n: Von allem wünsch' ich nichts, was immer sie bestrahle, Mich kann die weite Welt mit keiner Gab' erfreu'n.

Jenseits der Grenzen doch, die dies ihr Rund umfassen, Wo wahrer Sonne Licht durch andre Himmel scheint; Könnt' ich, der Hülle los, den Leib der Erde lassen: Vielleicht dort würd' ich schau'n, was hier mein Traum gemeint.

Dort labt' ich mich am Quell, nach welchem ist mein Schmachten, Dort reichte Hoffnung neu, dort Liebe mir die Hand, Dort fänd' ich jenes Gut, nach dem die Seelen trachten, Und das kein Name nennt in diesem Erdenland. 
Oh dürft' ich auf zu dir, du mein Geheimnis fliegen, Du meiner Wünsche Ziel - auf, mit des Frühroths Strahl! Warum muß ich verbannt hier auf der Erde liegen?

Ich habe nichts gemein mit diesem Jammertal!

Wann welk der Bäume Blatt fällt auf der Wiesenmatte, Erhebt der Nachtwind sich, der es dem Thal entreißt; Auch ich, auch ich bin gleich dem abgefallnen Blatte: Entführe, wilder Sturm, gleich ihm auch meinen Geist! ${ }^{1}$

\section{L'Isolement}

Souvent sur la montagne, à l'ombre du vieux chêne, Au coucher du soleil, tristement je m'assieds; Je promène au hasard mes regards sur la plaine, Dont le tableau changeant se déroule à mes pieds.

Ici, gronde le fleuve aux vagues écumantes, Il serpente, et s'enfonce en un lointain obscur; Là, le lac immobile étend ses eaux dormantes Où l'étoile du soir se lève dans l'azur.

Au sommet de ces monts couronnés de bois sombres, Le crépuscule encor jette un dernier rayon, Et le char vaporeux de la reine des ombres Monte, et blanchit déjà les bords de l'horizon.

Cependant, s'élançant de la flèche gothique, Un son religieux se répand dans les airs, Le voyageur s'arrête, et la cloche rustique Aux derniers bruits du jour mêle de saints concerts.

Mais à ces doux tableaux mon âme indifférente N'éprouve devant eux ni charme, ni transports, Je contemple la terre, ainsi qu'une ombre errante: Le soleil des vivants n'échauffe plus les morts.

De colline en colline en vain portant la vue, Du sud à l'Aquilon, de l'Aurore au couchant, Je parcours tous les points de l'immense étendue, Et je dis: Nulle part le bonheur ne m’attend.

1 Lamartine, Alphonse de: Auserlesene Gedichte. Metrisch übersetzt von Gustav Schwab. Mit beigefügtem französischen Texte. Stuttgart und Tübingen 1826, S. 3-7. 
Que me font ces vallons, ces palais, ces chaumières?

Vains objets dont pour moi le charme est envolé;

Fleuves, rochers, forêts, solitudes si chers,

Un seul être vous manque, et tout est dépeuplé.

Que le tour du soleil ou commence ou s'achève, D’un œil indifférent je le suis dans son cours;

En un ciel sombre ou pur qu'il se couche ou se lève, Qu'importe le soleil? je n'attends rien des jours.

Quand je pourrais le suivre en sa vaste carrière, Mes yeux verraient partout le vide et les déserts; Je ne désire rien de tout ce qu'il éclaire, Je ne demande rien, à l'immense univers.

Mais peut-être au-delà des bornes de sa sphère, Lieux où le vrai soleil éclaire d'autres cieux, $\mathrm{Si}$ je pouvais laisser ma dépouille à la terre, Ce que j’ai tant rêvé paraîtrait à mes yeux?

Là, je m'enivrerais à la source où j’aspire, Là, je retrouverais et l'espoir et l'amour, Et ce bien idéal que toute âme désire, Et qui n’a pas de nom au terrestre séjour!

Que ne puis-je, porté sur le char de l'Aurore, Vague objet de mes vœux, m'élancer jusqu'à toi, Sur la terre d'exil pourquoi resté-je encore?

Il n'est rien de commun entre la terre et moi.

Quand la feuille des bois tombe dans la prairie, Le vent du soir s'élève et l'arrache aux vallons;

Et moi, je suis semblable à la feuille flétrie:

Emportez-moi comme elle, orageux Aquilon!

In der nachfolgenden Interpretation stützte ich mich auf das französische Original. Mit diesem heute noch jedem französischen Schüler und jeder französischen Schülerin wenn nicht vertrauten, so doch bekannten Poem gelingt Alphonse de Lamartine eine dichterische Schöpfung, die zu den großen Leistungen der französischen Romantik zählt. Es handelt sich um ein Gedicht in Alexandrinern mit einem durchaus klassischen Strophenaufbau in insgesamt dreizehn „quatrains“. Dass es sich um ,klassische“ Alexandriner in einer klassischen Gedichtform handelt, zeigt schon die Übereinstimmung von Strophengrenzen und Satzgrenzen an. 
Lamartine griff auf einen Kreuzreim zurück, also „rimes croisées“ im Schema $a b a b$, wobei $a$ stets weiblich und $b$ stets männlich ist. Die Gesamtstruktur des Gedichts lässt sich relativ leicht erkennen, denn ein „Mais“ trennt zu Beginn der fünften Strophe einen ersten Teil ab und zu Beginn der zehnten Strophe einen dritten und letzten Teil, so dass der erste Teil des Gedichts vier, der zweite fünf und der dritte wiederum vier Strophen umfasst. Die zentrale Strophe ist daher die siebte Strophe, der also eine überragende Bedeutung aufgrund dieser Zentralstellung zukommt.

So verwundert auch nicht, dass gerade diese Strophe jenen Vers enthält, der zweifellos zu den meistzitierten Versen der Romantik zählt: „Un seul être vous manque, et tout est dépeuplé“. Dieser poetische Satz ist oft leicht abgewandelt worden, etwa von Jean Giraudoux in La Guerre de Troie n'aura pas lieu in ein „et tout est repeuplé“, oder - das ist wohl eines der neuesten Beispiele - von Marie Darrieussecq. Letztere hat in ihrem Erfolgsroman Truismes eine Fernsehsendung, in der nach verschollenen Menschen gesucht wird, mit dem Titel „Un seul être vous manque“ ironisch überschrieben. Wir bewegen uns also auf bekanntem literarischem Terrain, das ebenso zitierfähig wie gut memorierbar ist und sich daher für Persiflagen hervorragend eignet. Ich verweise an dieser Stelle im Übrigen auch auf die aufschlussreiche sozio-semiotische Interpretation des Gedichts durch Erich Köhler, der 1981, also vor fast vier Jahrzehnten, vor allem von den phonematischen Strukturen und Lexem-Rekurrenzen her seine Analyse dieses Textes aus vornahm ${ }^{2}$ und in seiner Vorlesung das Gedicht mit unverwechselbar schwäbischem Akzent vortrug.

Der Titel L'Isolement nimmt sich zweifellos ein in der Romantik häufig gewähltes Thema vor, das der Einsamkeit und mehr noch Vereinzelung des lyrischen Ich, das sich in mehrfachem Sinne in eine tiefe Einsamkeit, eine Isolierung zurückgeworfen sieht. Daher rührt auch der Titel der zeitnahen deutschen Übersetzung, die freilich etwas platter wirkt als das französische „Isolement“, in welchem die einsame Insel mitschwingt. Erich Köhler hat dies zugleich als Entfremdung aufgefasst und hiervon ausgehend Rückschlüsse auf die Klassenzugehörigkeit Lamartines zum verarmten Landadel gezogen, dem bekanntlich eine Vielzahl der französischen Frühromantiker angehörte. $\mathrm{Zu}$ ihnen zählen neben Lamartine unter anderem der Vicomte de Chateaubriand oder Bernardin de Saint-Pierre, aber auch Alfred de Musset und Alfred de Vigny sowie viele andere französische Poeten. Erich Köhler zeigte aber auch auf, wie sich ausgehend vom

2 Vgl. Köhler, Erich: Alphonse de Lamartine: „L’Isolement“. Versuch einer sozio-semiotischen Interpretation. In: Romanistische Zeitschrift für Literaturgeschichte (Heidelberg) 1-2 (1981), S. 129150. 
Titel verschiedene klangliche Sequenzen quer durch das Gedicht erstrecken. Man könnte von einem wahren Klangfächer sprechen, insoweit der Titel L'Isolement zentrale phonische Elemente, die das gesamte Gedicht strukturieren, vor uns von Beginn an paratextuell ausbreitet. Doch kann ich an dieser Stelle die Analyse der Phonotextualität, also der Klang-Text-Relationen, nicht wesentlich weiter vorwärtstreiben.

Sehen wir uns zunächst einmal die ersten vier Strophen des Gedichts und damit seinen ersten Teil genauer an! Die Situierung des Gedichts beginnt mit dem Verweis auf die Berge, ein Gebirge, wo sich das lyrische Ich oft unter einer alten Eiche niederzulassen pflegt. An dieser Stelle mögen wir uns mit dem kurzen und knappen Hinweis begnügen, dass die Spannung zwischen Individuum und Natur, Ich und Landschaft konstitutiv ist für die Herausbildung ebenso des modernen Subjekts als auch der romantischen Landschaft - eine Relation, die wir in unserer Vorlesung schon mehrfach erkundet haben. Beide bedingen sich wechselseitig: Die Landschaft ist ohne das romantische Subjekt schlechterdings nicht vorstellbar, und das Subjekt zieht seine Existenz aus dem konstruktiven Kontrast zur umgebenden Landschaft, mit der es in einer Korrespondenzbeziehung steht. Die philosophischen Voraussetzungen hierfür haben wir mit dem Verweis auf Ritter beziehungsweise Fichte kurz gestreift und wir sollten sie noch in diesem Gedicht wiederfinden, dessen Landschaft selbst dann nicht auf das Ich verzichten kann, wenn sie ihm, wie wir noch sehen werden, völlig indifferent geworden ist.

Was sich daran anschließt, ist nicht weniger topisch: Denn es folgt der Blick von oben, von den Bergen hinab in die Täler und Ebenen. Ein Blick von oben, den man auch als Schweben über den Dingen bezeichnen könnte und in welchem wir die in dieser Vorlesung bereits besprochene, von Jean-Jacques Rousseau ausgehende Suche nach Transparenz, Klarheit und Durchsichtigkeit erkennen können. Im zweiten Vers wird ebenfalls ein - nicht nur in der Lyrik - topisches Element des romantischen Diskurses eingeführt; der Sonnenuntergang nämlich, der auf die Szenerie ein langsam verlöschendes Licht wirft und damit von Beginn an die gesamte lyrische Inszenierung in ein melancholisches Dämmern taucht.

Dies deutet sich bereits im Wörtchen „tristement“ an, mit dem sich das lyrische Ich unter die alte Eiche setzt. Zu Füßen des lyrischen Ich zeigt sich nun ein Gemälde, freilich ein mobiles, sich wandelndes Gemälde, ein „tableau changeant“, das von Erich Köhler in seiner Interpretation übrigens erstaunlicherweise gar nicht gedeutet wurde, das uns aber vielleicht aus der Perspektive unserer Vorlesung auf die ständige Veränderung aller Dinge hinweist - und werden sie auch aus großer Höhe betrachtet. Vor allem aber entsteht in diesem Mobile der Landschaft eine Korrespondenz-Natur, in welcher wir deutlich eine Landschaft der Theorie erkennen können. Dabei spielt der Zufall, mit dem das Ich seine Blicke in die Ferne, auf die Ebene richtet, durchaus eine nicht zu unterschätzende Rolle. 
Das Ich jedenfalls befindet sich in einer erhobenen Position: Wir könnten daher mit dem angedeuteten Ausdruck des Erhabenen, des Sublimen rechnen. Doch diese an das Topische geknüpfte Erwartungshaltung wird enttäuscht und die mit ihr verbundene Theorie der Landschaft negiert.

Dies geschieht nicht von Beginn an, sondern erst im zweiten Teil des Gedichts. Zunächst erfolgt eine Verräumlichung und Konkretisierung des sich bewegenden Gemäldes, das nun in eine Abfolge sprachlicher Bilder - und zugleich auch Klangbilder - gebracht wird. Das Verschwinden des Flusses in einem weit entfernten Dunklen mag nicht nur die Reise und Bewegung des Lebens versinnbildlichen, sondern zugleich auch prophetisch ein dunkles Licht auf dessen weiteren Verlauf werfen: von der schäumenden, erregten Bewegtheit bis hin zu einem fernen, sachten Verschwinden und Sich-Auflösen.

Die zweite Hälfte des zweiten Verses, von „Ici“ und „Là“ klar strukturiert und durch die Interpunktion gegliedert, wird dem bewegten Wasser das ruhige Wasser eines Sees entgegengesetzt; ein für die Romantik typischer Kontrast also, der dieses Landschafts-Bild von Beginn an prägt. Darüber erhebt sich der Abendstern auf einem dunkelblauen Himmel, der sich im See spiegelt; ein Reflex des gesamten Kosmos, der auf der Spiegelfläche des stehenden, ruhigen Gewässers eingefangen wird. Wir könnten in der Tat glauben, es mit einem geradezu idealtypischen Gedicht zu tun zu haben - und in der Tat konnte die Forschung belegen, wie viele direkte Quellen diesen Fluss und See speisen, wie viele Formulierungen etwa auf Chateaubriand, Benjamin Constant oder andere vornehmlich französische Dichter verweisen.

Die dritte Strophe beginnt dort, wo bereits die erste Strophe eingesetzt hatte: nämlich auf der Spitze der Berge, also aus der Position des Erhabenen blickend in die letzten Strahlen des Sonnenuntergangs. Dies kann uns nur insoweit beeindrucken, als hier - zumindest auf Inhaltsebene - eine erstaunliche Rekurrenz stattfindet, so als könnte sich das lyrische Ich nicht mehr von dieser Szenerie trennen, die ihm jedoch auf mysteriöse Weise unzugänglich geworden ist. Und eben dies, so werden wir noch sehen, ist der Fall: Denn mit Sigmund Freud zu sprechen, wird das Heimelige dem Ich sukzessive unheimlich. Schon folgt nach dem Abendstern die Königin der Nacht, der im Französischen weibliche Mond, der nun alles in ein fahles, weißliches Licht taucht. Es fehlt kaum etwas zur romantischen Szenerie, die der Dichter in diesen Versen für uns entwirft.

Bereits die vierte Strophe deutet jedoch an, dass sich etwas anderes in diesem Bild eingenistet hat: eine Ahnung, die durch die Rekurrenz aufkam, nun aber durch das eröffnende „Cependant“ der vierten Strophe konkrete Gestalt annimmt. An dieser Stelle des Gedichts erscheint nun die gotische Kirchturmspitze und damit ein Element okzidentaler Kultur. Das gotische Himmelstreben zu Gott ist eine Verbeugung vor dem christlichen Abendland und zugleich eine Wertschät- 
zung des in der Romantik stark aufgewerteten Mittelalters, das mit seiner Frömmigkeit eingeblendet wird. Die Lüfte transportieren den Schall dieser Kirchenglocke, deren Klang eine Art Rufen aus der Ferne, vielleicht aus zu großer Ferne darstellt. Die Figur des Reisenden hält in ihrer Bewegung inne, wird durch den Klang also an andere Aufgaben und Lebensinhalte erinnert und an eine Sammlung, eine Versammlung, eine religiöse Gemeinschaft gemahnt, die sich nun am Ort der Kultur inmitten einer Szenerie der Schöpfung bildet. Die Bewegung des Wanderers erstarrt und mit ihm die Mobilität der Landschaft, die er durchquert und deren Horizonte und Perspektiven sich ständig veränderten. Der Tag und mit ihm das Tagewerk geht zu Ende, es bricht eine Zeit der Transzendenz an, in der die Nacht an die Stelle des Tages rückt. Der Reisende kann seine Bewegung nicht fortführen und kehrt wohl in dem rustikalen Ort mit seiner mittelalterlichen Vergangenheit ein: Das sakrale Element beherrscht den Ausgang dieses ersten Teils mit seiner klanglichen Orchestrierung.

Die fünfte Strophe baut nun den ersten scharfen Kontrast, den ersten direkten Gegensatz auf zwischen den „doux tableaux“, welche die vorangehenden Strophen entwerfen, und der indifferenten Seele des lyrischen Ich, die sich von den zuvor entworfenen romantischen Naturbildern nicht affizieren lässt und ihnen gegenüber indifferent, unbeeindruckt bleibt. Weder „charme“ noch „transport“, weder Anziehungskraft noch Verzauberung kann diese sich unvermittelt auftuende Distanz und Indifferenz durchbrechen. Die Kontemplation der Erde, der „terre“ führt gerade nicht zur Transzendenz, zum Lob der Schöpfung, zur Erkenntnis des Guten, Schönen und Ewigen, sondern hinterlässt die „ombre errante“, die nicht in die Stille und Kontemplation der Versammlung überführt werden kann.

In die Gegensatzposition zur Sonne der Lebenden begibt sich in dieser poetischen Wendung der Zustand der Toten, genauer noch jener Menschen und jenes Menschen, der zu den lebendigen Toten zählt. Das Ich ist offensichtlich allem Irdischen bereits fremd, ist von diesem isoliert, abgeschieden, vereinzelt: ein der Welt entfremdetes modernes Subjekt, isoliert und damit insularisiert von allem. Nichts kann mehr den Blick des lyrischen Ich befriedigen, der die verschiedenen Hügel umherirrend streift, ohne doch einen festen Halt, eine Erkenntnis zu finden: eine klare Antwort zu formulieren auf die zuvor entworfene Landschaft der Theorie mit ihrem Schöpfungsglück. In allen Himmelsrichtungen, die auch vom Nordwind verkörpert werden, kann das Ich nichts wirklich für sich erblicken. Wir werden bei Arthur Rimbaud erneut auf diesen „Aquilon“ stoßen, den Nordwind und dessen Umdeutung innerhalb einer Lyrik, die der Romantik endgültig entzogen ist.

Nirgendwo, „Nulle part“, an keinem Ort warte das Glück auf ihn: Alles sei leer und ohne Glücksversprechen. Diese Negation eröffnet zugleich - wenn Sie 
den Satz anders lesen, indem sie für „Nulle part“ einfach U-Topia einsetzen - eine letzte Hoffnung, die allein noch im Utopischen liegen kann: kein Ort, nirgends. Damit aber wird angesichts der Unermesslichkeit des Raumes, den das Ich überblickt, zugleich deutlich, dass dieser Raum sehr weit weg liegen muss oder - wie Baudelaire später formulieren sollte - Anywhere out of the world. Eine zeitliche Grundstruktur des Gedichts wird damit erkennbar, insoweit der erste Teil der Vergangenheit, der zweite Teil einer nicht mehr erlösenden Gegenwart und der dritte Teil einer Zukunft überantwortet wird, welche freilich die vagen Hoffnungen noch nicht zu konkretisieren vermag. Nirgendwo aber ist das fragwürdig gewordene Subjekt zu Hause!

Die direkte Rede des lyrischen Ich, die von der ersten Person Singular selbst eingeführt und damit zugleich verstärkt wird, intensiviert die abwertende, enttäuschte Betrachtung all jener Gegenstände, die doch in ihm Bewunderung, zumindest aber eine gewisse Erfülltheit hätten provozieren müssen. Doch alles ist zu „Vaines objets“ geworden, zu Objekten, die für das Ich eine Leere beinhalten, gerade weil sich im Inneren des Ich eine solche aufgetan hat, welche nicht mehr mit Sinn zu füllen ist. Alles, was einen Wert besaß, wohl auch für das lyrische Ich, das von seinen „solitudes si chers“ spricht, hat völlig an Wert verloren und versinkt in einer simplen Aufzählung, einem aneinandergereihten Katalog von Gegenständen, die zugleich auch immer schon Gegenstände der lyrischen Dichtkunst der Romantik waren.

Erst an dieser wohlvorbereiteten Stelle kommt jener Vers erst ganz zum Tragen, welcher der Präsenz aller Objekte, aller Gegenstände, die Absenz eines einzigen Objekts, des Objekts der Liebe und des Begehrens, entgegenstellt. Die Absenz entzaubert die Präsenz als für das Ich nur scheinbare. Dem Sein gewesener Dinge wird das Nicht-Sein eines wirklichen Seins entgegengestellt, eines „seul être“, das die Überfülle aus einer einzigen Leere heraus schlagartig entvölkert. Dabei hat Erich Köhler in seiner Deutung von L'Isolement mit Recht darauf hingewiesen, dass das „,vous“ im Vers „Un seul être vous“ manque deutlich daran gemahnt, dass es hier nicht nur um eine individuelle, sondern zugleich und nicht weniger um eine kollektive Dimension geht. Die gesellschaftliche Dimension ist folglich keineswegs durch die Vereinzelung und Einsamkeit des Ich ausgeschlossen.

Auf diese kunstvolle Weise steht also der „manque“, das Fehlen inmitten der Überfülle, die nun zum reinen Dekor herabgewürdigt ist. So steht also die Absenz in der Präsenz im Zentrum des Gedichts. Es mag sein, dass wir es hier mit der den Poststrukturalisten so teuren Leere im Zentrum zu tun haben; doch es ist keine erwünschte Leere, kein leeres Zentrum, wie dies später die Postmoderne proklamieren sollte. Zugleich ergibt sich an dieser Stelle ein Kritikpunkt an Winfried Wehles Leopardi-Deutung, die wir im entsprechenden Kapitel herangezo- 
gen hatten: Denn leeres Zentrum ist nicht gleich leeres Zentrum. Hier geht es um eine Leere, welche ihre Bewohner abstößt, ja sogar anekelt. Sie hinterlässt den „ennui“, das Unausgefüllt-Sein, das doch stets nach Ausgefüllt-Werden, nach neuem Sinn strebt.

Auch die Bewegungen des Kosmos, der Lauf der Sonne, sind nun dem Ich völlig indifferent geworden. Es folgt zwar noch dieser Bewegung, doch bietet diese keinerlei kosmische Zuflucht, kein Aufgehoben-Sein in einer totalen Schöpfung voller Ordnung und Schönheit mehr. Alles ist in-different geworden, weil Differenzen angesichts des zu konstatierenden Verlusts eines Wesens, des geliebten Wesens, keine Rolle mehr spielen. Die Indifferenz und die damit verbundene Weltflucht ist die Folge dieses Weltschmerzes, der zweifellos romantische Züge trägt. Nichts mehr, keine Hoffnung mehr wird vom nächsten Tag, von den Tagen von Seiten des lyrischen ich erwartet: Alles ist gleichgültig und mehr noch gleichungültig geworden, mag die Sonne nun auf- oder untergehen!

Auch an dieser Stelle wird das ,attendre“, also die ,attente“ noch in der negativen Formulierung erkennbar. Alle Erwartungen des einst hoffnungsfrohen Ich sind nicht verschwunden, sondern ins Negative verkehrt. Überall auf der Erde, wohin das Ich der Sonne folgen könnte, würde es doch nur Leere und Wüsten antreffen. Und im Begriff der Wüste - wir erinnern uns an Chateaubriand, aber auch an Sarmiento - manifestiert sich noch einmal das „dépeuplé“: Alles ist entvölkert, der ganze Planet ist menschenleer; da helfen keine Gegen-Stände, keine Ob-jekte, die das Sub-jekt in die Präsenz rufen könnten.

Aus dem Subjekt heraus alleine lässt sich keine neue Sinngebung finden, ebenso wenig wie aus einer zum Topos, zum Ort erstarrten Natur. Das Ich erklärt, auf jegliches Streben, auf jegliches Begehren längst verzichtet zu haben, sich nichts mehr von diesem „,vaste univers“ zu erhoffen, das zu einem entvölkerten Planeten geworden ist. Wir spüren, wie nahe wir hier dem Bild einer Apokalypse sind: nicht einer der Explosion oder Aggression, sondern einer Apokalypse der Implosion; also genau das, wofür das „mal du siècle“, wofür die Melancholie steht.

Damit sind wir nun an das Ende des zweiten Teils gelangt und nähern uns dem dritten und letzten Teil: Besitzt diese Makrostruktur nun die Form eines Syllogismus oder doch, wie Erich Köhler in seiner marxistisch fundierten Analyse meinte, die Form eines dialektischen Dreischritts von These, Antithese und Synthese? Wir müssen uns zur Beantwortung dieser Frage diesem letzten Abschnitt zuwenden, der mit dem „Mais“ der zehnten und damit erneut mit einem scharfen Kontrast, einem glatten Widerspruch und Gegensatz einsetzt.

Nach diesem „Aber“ erscheint nun noch eine allerletzte Hoffnung; und diese Hoffnung wird räumlich wie zeitlich in ein Jenseits, ein ,au-delà“ projiziert, jenseits aller Grenzen. Zugleich wird es unter die Kautele eines „Vielleicht“ gestellt, 
folglich in der Sphäre des „peut-être“ angesiedelt. Es ist diejenige des Anderen, die sich auftut: Andere Himmel gibt es hier, die über eine wahre Sonne verfügen eine Alterität als Authentizität. Der Himmel signalisiert freilich ein anderes, nicht mehr irdisches Leben, dem auch die Wortwahl entspricht: Denn die irdische Hülle ist es, die das lyrische Ich auf der Erde zurücklassen will; und diese Erde wird zu einer „terre d'exil“ in der zweitletzten Strophe. Damit ist mehr gemeint, als dass wir nur Gast auf Erden wären und uns hier gleichsam in einem christlichen Sinne lediglich in der Verbannung, in einem Exil befänden, das uns in seiner heilsgeschichtlichen Bedeutung an die Zeiten Israels gemahnt. Erich Köhler hat in seiner Deutung des Gedichts sehr schön die Klang- und Lexem-Rekurrenzen herausgearbeitet und gezeigt, wie sich davon ausgehend die unterschiedlichsten Ebenen in der Wortfügung „terre d'exil“ kreuzen. Dies muss an dieser Stelle unserer eigenen Deutung nicht wiederholt werden.

Die elfte Strophe zeigt mit aller Deutlichkeit auf, dass das lyrische Ich noch weit davon entfernt ist, alles Streben, alles Hoffen aufzugeben. Denn es sehnt sich noch immer nach der wahren Quelle, der wahren Hoffnung, der wahren Liebe, die im Übrigen auch nicht gefunden, sondern wiedergefunden werden sollen. Es gibt also so etwas wie eine Rückkehr zu einer Wahrheit, die von einer einzigen Quelle ausgeht - und in diesem Zusammenhang schwingt nun deutlich neuplatonisches Gedankengut mit. Es ist ein dem Christentum durchaus entrücktes Ideal, das sich in jenem „bien idéal“ ausdrückt, nach dem jedermann strebe; eine ideelle Größe, die sich nicht einfach mit dem Christengott gleichsetzen lässt.

Denn gerade die Rede vom „Exil“ scheint mir eine alttestamentarische, in gewisser Weise sogar heidnische Dimension einzublenden, die freilich ganz im Sinne der literatursoziologischen Deutung Köhlers durch die aktuelle gesellschaftliche Dimension zu ergänzen wäre. Denn nicht umsonst ist gerade jene Gruppe französischer Lyriker der Frühromantik von einem Funktionslos-Werden ihrer eigenen Klasse - im Falle Lamartines also vom niederen Landadel betroffen, befindet sich also in einer Art geistigem Exil, das im Übrigen andere aristokratische Gruppen von Beginn der Französischen Revolution an ereilt hatte - verwiesen sei hier nur auf den von uns erläuterten Fall Chateaubriand. Entscheidend ist, so scheint mir, dass das Exil stets ein Anderswo impliziert, welches zugleich auch das „Nulle part“ des zweiten Teils in Lamartines Gedicht dementiert.

Die Isotopien des Fremdwerdens, der Isolierung sind also weitaus größer und umfassender als die in der Forschungsliteratur so häufig zu findende Behauptung, das Gedicht stelle die lyrische Verarbeitung des Todes von Lamartines tuberkulosekranker Geliebter Julie Charles dar. Es ist die Namenlosigkeit des idealen und ideellen Guten, um das dieses Gedicht kreist; und damit (ver)ortet es in der Sprache selbst eine Leere, die es umkreist und die es letztlich doch wieder zu 
füllen versucht. So führt das Gedicht selbst die Problematik der Leere auf Ebene der von ihm verwendeten Sprache vor: Auch hier fehlt nur ein einzig Wesen, und alles ist entvölkert.

Dann freilich folgt eine direkte Anrede an ein Du, das zum Ziel der Träume des Ich geworden ist, welches von Beginn an in einer großen Höhe situiert wird, sich aber nun erst von dort in kosmische Dimensionen aufzuschwingen sucht. So sind denn doch die traditionelle Topik und Metaphorik, die Aufwertung der Höhe und die Betonung des Lichts wieder sichergestellt. Mit seinen Alexandrinern und seiner Strophen- und Satzgliederung bewegt sich L'Isolement durchaus noch innerhalb traditioneller, ja klassischer Vorbilder. Das Gedicht ist zweifelsohne kein revolutionäres Gedicht, es stellt nur eine aktuelle Position und letztlich auch eine gesellschaftlich-historische Situation als ausweglos dar.

Dabei unternimmt es zugleich eine Kritik der frühen Formensprache der Romantik, indem es deren Versatzstücke katalogisiert und allesamt verwirft. Denn hinter allem schimmert noch immer die Leere durch, in allem zeigt sich noch immer das Irdische, das alles zum Scheitern verdammt und kommunikationslos werden lässt. Hier aber, in der zwölften Strophe, wird der Bogen, wird der Kreis geschlossen und eine direkte Kommunikation mit einem $\mathrm{Du}$ angestrebt, das allerdings ebenso namenlos bleibt wie das „bien idéal“. Daraus ergibt sich eine radikale Trennung zwischen dem Ich und der Erde, die gleichsam zum Schlusspunkt der zwölften Strophe wird. Dergestalt formt sich eine Schlussfolgerung, die den gesamten Gedankengang abschließt und krönt.

Und doch öffnet sich das Gedicht noch ein letztes Mal zu einer allerletzten, einer dreizehnten Strophe, was durchaus an Gérard de Nervals „La treizième revient, et c'est toujours la premiére ....“ erinnert. Denn nun wird alles in eine Metaphorik der Jahreszeiten, die bekanntlich zirkulär sind und stets wiederkehren, in eine Metaphorik des Herbstes (die stets auch ein Lebensalter anzeigt) und des Blattes (das nicht nur die Jahreszeiten und ein Lebensende, sondern zugleich auch topisch das Schreiben assoziiert) übertragen. Das Ich fühlt sich einem Blatte im Wind ähnlich (,semblable“); und es stellt sich die Frage, ob wir an dieser Stelle nur eine topische Wiederanknüpfung an eine Sprache der Romantik erkennen dürfen, welche doch gerade hinterfragt worden war.

Erich Köhler, mit dessen Deutung wir in diesem Kapitel ein fortgesetztes Gespräch führen durften, hat vorsichtshalber diese Dimension weggeblendet und lässt das Gedicht in der „terre d'exil“ gipfeln. Ist diese dreizehnte Strophe also überflüssig? Dient sie nur dazu, dem Gedicht eine zentrale, eben die siebte Strophe zu verschaffen, von deren Ende aus sich alle Isotopien, alle Bedeutungsebenen erschließen? Ich glaube nicht! Wir können Alphonse de Lamartine als herausragendem Schriftsteller sicherlich soweit vertrauen, dass er nicht eine völlig überflüssige Strophe an das Ende seiner wohl berühmtesten Schöpfung 
gestellt hätte. Er hätte sie sicherlich gestrichen, wäre sie ihm am Ende als überzählig erschienen.

Wenn diese Strophe am Ende von L'Isolement aber nicht überflüssig ist, in welchen Funktionszusammenhang ließe sie sich dann stellen? Sollte das Gedicht nur auf ein Hinweggefegt-Werden durch den Nordwind, durch den „Aquilon“ hinauslaufen? Und was ist unter diesem im Gedicht rekurrenten „Aquilon“ überhaupt zu verstehen?

Bei Arthur Rimbaud wird er ein gutes halbes Jahrhundert später im Gedicht Qu'est-ce pour nous mon coeur als „et l'Aquilon encor sur les débris“ erscheinen, als der Nordwind, der von Preußen her nach dem Desaster der Pariser Kommune die französische Hauptstadt und Frankreich mit seiner Gewalt überzieht und ein Trauma des Panlatinismus schaffen wird. Denn angesichts seiner Wucht und Gewalt sind die Völker des lateinischen Europa, die Völker der lateinischen Welt, so scheint es, schutzlos dem Ansturm der Germanen preisgegeben, welche der „Aquilon“ bei Rimbaud verkörpert. Doch noch stehen wir nicht im Schatten jener scheinbaren Dekadenz der lateinischen Völker, welche dem Ansturm der Germanen wie der Angelsachsen an allen Fronten weichen müssen und die - wie Rubén Daríos Gedicht $A$ Roosevelt es ausdrückte - vielleicht bald schon dem Untergang geweiht sind: Noch ist bei Alphonse de Lamartine der Duft der finisekulären Dekadenz, die Schwüle des Fin de siècle weit entfernt.

Denn so kann es Lamartine am Ende des zweiten Jahrzehnts des 19. Jahrhunderts nicht gemeint haben! Soeben erst hatten noch die französischen Heere unter Napoleon ganz Europa in Furcht und Schrecken versetzt: Europa erzitterte unter dem Stiefel der französischen Truppen und brauchte noch lange, um sich von der Franzosenangst zu erholen. Erstaunlich aber, dass es hier der Nordwind ist, der diese Form der Romantik zugleich noch einmal aufbrausen lässt und langsam, sachte zum Verschwinden bringt. Sind es die enttäuschten Hoffnungen der Französischen Revolution, sind es die Ängste davor, unter dem Druck des Nordens, der alliierten Heere die alte Macht und Größe verlieren zu müssen und nach dem Verlust der Vormachtstellung fortan in einer „terre d'exil“ des eigenen Landes zu leben? Sind es gar die Ängste, von der geistigen Stärke des Nordens überrannt oder hinweggefegt zu werden, so wie die Romantik einen eher nördlichen Wind durch die Vorstellungswelt der romanischen Romantik brausen ließ?

Denken wir an dieser Stelle noch einmal zurück an unsere Untersuchungen zu Germaine de Staël und das Verhältnis zwischen dem französisch- und dem deutschsprachigen Europa! Auch Lamartine setzte sich in seiner Zeit als Diplomat und vor allem als Politiker für eine Überwindung des konfrontativen Gegensatzes zwischen einem noch immer nach der Nationenbildung suchenden Deutschland und dem Nationalstaat Frankreich ein. Die Pariser „Commune“ und den preußisch-französischen Krieg erlebte er freilich nicht mehr. 
Zweifellos nimmt die Blatt-Metaphorik den Baum des allerersten Verses dieses Gedichts wieder auf, wobei damit zugleich die Möglichkeit erscheinen könnte, dereinst wieder unter diesem Baum in vertrauter Umgebung sitzen zu können. Aber dies wäre pure Spekulation! Es mag sein, dass in diesem Zusammenhang eine andere Naturkonzeption hinzutritt, die nicht auf die Transparenz, nicht auf die „doux tableaux“, nicht auf die Ausgeglichenheit einer Landschaft als Locus amoenus ausgerichtet ist, sondern die Macht und Gewalt der Natur als bedrohlich, als gefährlich und gefährdend erscheinen lässt. Es handelt sich um eine Natur, die im Zeichen des Nordens steht, eines „ailleurs“, das zweifellos nicht das von Lamartine angestrebte Anderswo ist. Damit könnte an eben dieser exponierten Stelle eine andere Romantik-Konzeption Einzug in Lamartines Schaffen halten, die deutlich unter dem eindrücklichen Bild einer anderen Natur, einer Natur als Gefährdung, einer Natur des Nordens und nicht länger im Schatten einer „Pensée du Midi“ im Sinne Germaine de Staëls stünde.

Immerhin: Eine unbestreitbare Endzeiterwartung zeichnet sich durchaus im Nordwind des „Aquilon“ ab. Der Bezug zum lateinischen „aquilo“, dem Wind des Nordens, der so schnell für die Römer war wie der Flügelschlag des Adlers, könnte höchst ambivalent auf den Sturm von Norden her und damit auf eine künftige Vorherrschaft des Nordens verweisen wie auch auf jenen Sturm, den einst der französische Kaiseradler Napoleons ausgelöst und über Europa entfesselt hatte. Dieser von Napoleon entfachte Sturm hatte letztlich auch die französische Gesellschaft, die Lamartine und seiner sozialen Klasse zum Exil wurde, so grundlegend verändert, dass sie dem alten Landadel die eigene Funktionslosigkeit drastisch vor Augen führen musste.

Auf diese Weise könnte sich unter dem lateinischen „aquilo“ vielleicht doch noch immer der Adler (,aquila“) verbergen. Die Lexem-Rekurrenz im Gedicht spielt eine wichtige Rolle: Denn die Assoziation mit der Himmelsrichtung, wie sie beim ersten Auftauchen dieses Lexems im Gedicht gegeben ist, wird beim zweiten zweifellos durch die Seme des Windes und vor allem der Gewaltsamkeit ergänzt. An dieser Stelle könnte ein neues, von außen kommendes Denken des Nordens in die Welt Lamartines einbrechen, die von keinem christlichen Gott, von keinem mittelalterlichen Kirchturm mehr zusammengehalten werden kann. Die Gemeinschaft der Gläubigen, die Gemeinschaft auch des lokalen Dorfes unterliegt diesem gewalttätigen Wind des Nordens, der alles verändert und die Erde zum Exil der aus ihrem eigenen Land Vertriebenen werden lässt. Gleich einer „feuille flétrie“ wird das Ich im letzten Vers von L'Isolement davongetragen vom „orageux Aquilon“.

Lassen Sie uns abschließend zum einen festhalten, dass der Katalog romantischer Versatzstücke schon Alphonse de Lamartine zum Überdruss geworden ist! So zeigen sich selbst im Herzen eines jener Gedichte, die wie wenige andere für die 
europäische Romantik einstehen, nach einigen Jahrzehnten Verschleißerscheinungen romantischer Dichtung. Zugleich aber wird ein essentielles, fundamentales Fehlen, ein „manque“ sichtbar; eine Leere im Zentrum, die gefüllt werden muss. Diese Leere löst einen Impuls aus jener „terre“ aus, die dem lyrischen ich längst schon zum Exil, zu einem „séjour“ und nicht zur Heimstatt geworden ist.

Auf diese Weise ergibt sich in der Tat eine Prozessualität der Isolierung, der Vereinzelung, der Insularität und Entfremdung, die das Ich wiederum einer nicht mehr heilen Natur und auch einer nicht mehr heilenden Natur absolut gegenüberstellt. Das Ich gerät zur isolierten, vom Festland abgeschnittenen Insel, genauer: zu einer Insel-Welt, die für sich ist und ihre Fülle in der Abgeschlossenheit entfaltet. Der Sturm des Nordens, der Sturm der Moderne wird über diese Insel, die sich ihre kulturelle Fülle durchaus erhält, hinwegbrausen wie über jenen verarmten Landadel, dem der Dichter wie manch anderer französischer Poet der Romantik angehört.

Die intime Kommunikation mit einem Du ist zerbrochen; und so weitet sich die Leere, „le vide“ unentrinnbar aus, seien die Ebenen noch so unermesslich weit. Die Metaphorik des Flusses, die in ihrer Vektorizität und Linearität topisch für das Leben steht, hat am Ende in ihrer Bewegung schon jene andere Bewegung vorweggenommen, die das Abreißen der Blätter durch den Nordwind in der dreizehnten und letzten Strophe auszeichnet. Das „bien idéal“ ist auf dieser Erde nicht mehr zu gewinnen, und die Natur besitzt nicht länger die Kräfte, dieses erträumte Ideal in die Wirklichkeit umzusetzen. Sie erscheint entweder als die sanfte, gütige, bergende Natur oder als eine Natur, die alles mit sich fortreißt und letztlich die Insel vom Festland trennt. In dieser Konfiguration erscheint auch das Gedicht selbst: Ist es nicht, wie der karibische Dichter Derek Walcott einmal formulierte, eine Insel, die vom Festland weggebrochen ist? „Poetry is an island that breaks away from the main. “" ${ }^{3}$ So erklärt sich auch der Titel des Gedichts, das eben nicht Isolation, sondern L'Isolement lautet und damit jenen Prozess beleuchtet, der zur Gestaltung ebenso des lyrischen Ich wie der Gedicht-Werdung selbst führt.

Diese lyrische Isolierung und Vereinzelung erscheint zwar als durchaus ambivalent, grundlegende Hoffnung aber birgt dieses Isolement kaum mehr, sondern

3 Walcott, Derek: The Antilles, Fragments of Epic Memory. The 1992 Nobel Lecture. In: World Literature Today (Oklahoma) LXVII, 2 (Spring 1993), S. 261-267; hier zitiert nach Walcott, Derek: The Antilles: Fragments of epic Memory. In (ders.): What the Twilight Says. Essays. New York: Farrar, Straus and Giroux 1998, S. 70. Vgl. hierzu im Kontext insularer Epistemologie Ette, Ottmar: Von Inseln, Grenzen und Vektoren. Versuch über die fraktale Inselwelt der Karibik. In: Braig, Marianne / Ette, Ottmar / Ingenschay, Dieter / Maihold, Günther (Hg.): Grenzen der Macht - Macht der Grenzen. Lateinamerika im globalen Kontext, S. 135-180. 
nur noch eine immense Leere angesichts einer Lebenserfahrung, in welcher der Tod der geliebten Frau nicht rückgängig zu machen ist. So erscheint es auch als konsequent, wenn das Element dieser Leere, die Luft, der Wind, dieses Gedicht beendet und seine Blätter hinwegfegt und mit sich fortreißt. Dem Ich ist eine Kommunikation mit dem erhofften Du nicht mehr gelungen: Nur im Ideal noch erscheint diese direkte Kommunikation als eine Utopie, welche auf dieser Erde über keinen Ort mehr verfügt. Die anderen Himmel, die ,autres cieux“, sind nicht mehr von dieser Welt. Doch noch immer scheint die Utopie in der lyrischen Insel des Gedichts auf, das im Verständnis seiner Verfasserinnen und Verfasser, in der Deutung seiner Leserinnen und Leser weit über die Zeit und weit über den Raum hinausreicht. 